\title{
Active surveillance for prostate cancer
}

Prostate cancer management continues to rapidly evolve for men presenting with all stages of disease. In response to the pandemic of overdetection and overtreatment of indolent prostate cancer, active surveillance has become a standard management strategy for many men with low-risk cancers. Many challenges with clinical implementation of active surveillance persist, including optimal selection criteria, surveillance strategies, indicators of disease progression and when further treatment may be warranted.

As guest editors of this issue of Translational Andrology and Urology (TAU), we aimed to provide information on the contemporary practice of active surveillance for prostate cancer. We invited a number of experts and thought leaders to provide their perspectives on topics ranging from racial disparities, triggers for intervention and economic perspectives. Utilization of active surveillance is increasing; however, many barriers exist. Data from ongoing clinical trials will provide valuable insight for the care of these men while integrating novel technologies such as germline genetic risk, imaging and molecular biomarkers to safely offer this option to a greater number of men with improved outcomes. It is our hope to share contemporary knowledge regarding active surveillance for prostate cancer and broaden the number of men who might benefit from this approach.

\section{Acknowledgements}

None.

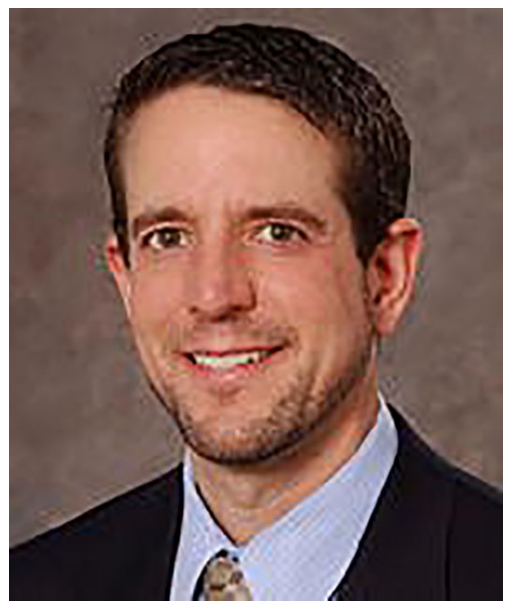

Marc A. Dall'Era

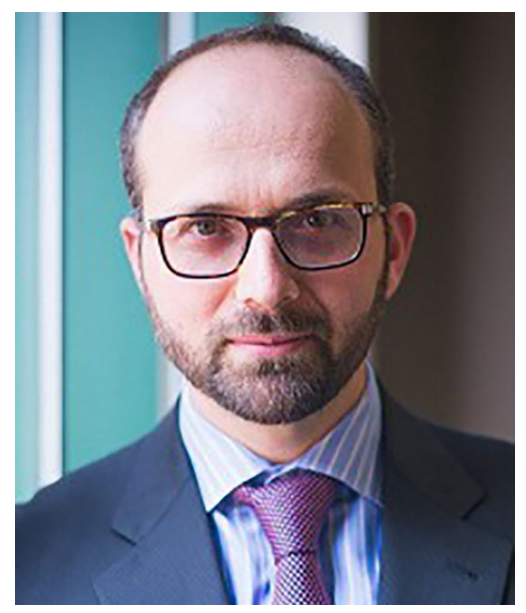

Benjamin J. Davies

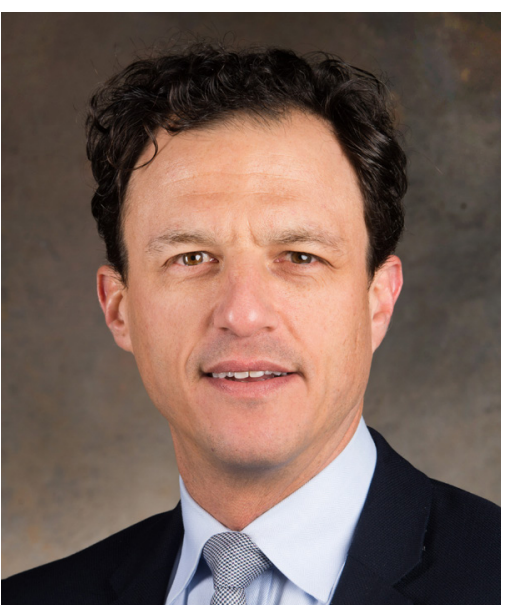

Scott Eggener 
Marc A. Dall'Era, MD Associate Professor and Vice Chair of Urology, University of California, Davis, School of Medicine, California, USA.

(Email: mdallera@ucdavis.edu) Benjamin J. Davies, MD Associate Professor of Urology, University of Pittsburgh School of Medicine, Pittsburgh, USA.

(Email: daviesbj@upmc.edu)

Scott Eggener, MD

Professor of Surgery; Director, Prostate Cancer Program; Director of Translational and Outcomes Research, Section of Urology, the University of Chicago Medicine, Chicago, USA. (Email: seggener@uchicago.edu) doi: $10.21037 /$ tau.2018.03.03

Conflicts of Interest: The authors have no conflicts of interest to declare.

View this article at: http://dx.doi.org/10.21037/tau.2018.03.03

Cite this article as: Dall'Era MA, Davies BJ, Eggener S. Active surveillance for prostate cancer. Transl Androl Urol 2018;7(2):195-196. doi: 10.21037/tau.2018.03.03 